\title{
DINÂMICA POPULACIONAL DE Saissetia oleae (HEMIPTERA: COCCIDAE) EM OLIVEIRAS ${ }^{1}$
}

\author{
GABRIELA CHESIM DE SOUZA², LUÍZA RODRIGUES REDAELLI ${ }^{3}$, \\ VERA REGINA DOS SANTOS WOLFF ${ }^{4}$
}

RESUMO - A olivicultura é uma atividade recente no Rio Grande do Sul, com poucas informações sobre os insetos associados. A dinâmica populacional de Saissetia oleae (Oliver) e seu parasitismo natural foram avaliados em pomar de Olea europaea L., cultivar Arbequina, mantidos sob manejo convencional, situado

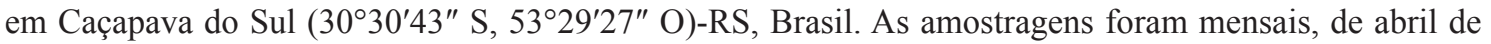
2012 a março de 2013. Em cada ocasião, em 20 plantas sorteadas, e dos quadrantes da copa (norte, sul, leste e oeste), foram retirados dois ramos aleatórios ( 20 a $30 \mathrm{~cm}$ de comprimento, com no mínimo 20 folhas), um interno e outro externo. A maior abundância de $S$. oleae foi na primavera e no verão, e a temperatura foi o fator que interferiu nesta dinâmica. Saissetia oleae distribuiu-se de forma uniforme entre os quadrantes e entre os ramos internos e externos da copa. Nas folhas, observou-se maior abundância de ninfas de primeiro e segundo instares, e nos ramos de ninfas de terceiro instar, adultos e adultos com ovos. A taxa de parasitismo foi de $3,40 \%$.

Termos para indexação: cochonilha-negra, dinâmica populacional, Olea europaea.

\section{POPULATION DYNAMICS OF Saissetia oleae (HEMIPTERA: COCCIDAE) ON OLIVE TREES}

\begin{abstract}
Oliviculture is a recent activity in Rio Grande do Sul with little information on associated insects. The population dynamics of Saissetia oleae (Oliver) and its parasitism was evaluated in olive orchard, Olea europaea L., cultivar Arbequina, kept under traditional management, situated in Caçapava do Sul $\left(30^{\circ} 30^{\prime} 43^{\prime \prime}\right.$ S, 5329'27" W), RS, Brazil. Samples were monthly collected from April 2012 to March 2013. At each occasion 20 trees were randomly selected and, from each quadrant of their canopies (northern, southern, eastern and western) two branches (20 - 30 cm - length and containingat least 20 leaves) were removed randomly, one inside and one outside the tree. The greater abundance of $S$. oleae was in the spring and summer and the temperature was the factor that interfered in this dynamic. Saissetia oleae was distributed in a uniform way between quadrant and between the internal and external branches. In the leaves were observed with greater abundance $S$. oleae of first and second nympal stages, while in the branches third instar nymphs, adults and adults with eggs. The parasitism rate was $3.40 \%$.
\end{abstract}

Index terms: black scale, population dynamics, Olea europaea.

${ }^{1}$ (Trabalho 213-14). Recebido em: 21-07-2014. Aceito para publicação em: 09-04-2015.

${ }^{2}$ Eng. Agr., Mestre da UFRGS - PPG Fitotecnia, Departamento de Fitossanidade. E-mail: gabycsouza@gmail.com

${ }^{3}$ Eng. Agr., Dr., Professora da UFRGS - PPG Fitotecnia, Departamento de Fitossanidade. E-mail: 1uredael@ufrgs.br

${ }^{4}$ Biól. Dr., Pesquisadora da FEPAGRO - Unidade Central Porto Alegre. E-mail: wolffvera@gmail.com 


\section{INTRODUÇ̃̃O}

Saissetia oleae (Oliver) (Hemiptera: Coccidae), também conhecida como cochonilhapreta, cochonilha-da-oliveira ou ainda cochonilhanegra, está distribuída em todas as regiões tropicais e subtropicais do mundo, considerada como praga nas culturas dos citros e de oliveiras (MURÚA; FIDALGO, 2001). Os danos diretos causados em oliveiras ocorrem pela sucção da seiva, porém os indiretos são mais comuns, como o desenvolvimento da fumagina, fungo de coloração negra, que se mantém através do exsudato açucarado (honeydew) liberado pela praga. Este fungo forma uma grossa camada que impede a passagem de luz, prejudicando a respiração e a produção de fotoassimilados, provocando a depressão da planta, diminuindo sua brotação e, consequentemente, reduzindo a produção (BARRANCO et al., 2008).

No Brasil, em Minas Gerais, Prado et al. (2012) constataram que $S$. oleae foi a espécie mais abundante em oliveiras, apontada como praga primária na cultura (SANTA-CECÍLIA et al., 2014). As fêmeas de $S$. oleae são de coloração marromescura, medem de 3 a $4 \mathrm{~mm}$, têm corpo em formato de "concha" com uma rugosidade em forma de "H". As ninfas de primeiro e segundo instares são de coloração amarelo-clara a parda (BARRANCO et al., 2008) e medem de 0,4 a 1,5 mm (PRADO et al., 2012). As de terceiro têm carapaça amarelada, e as elevações em forma de "H" já são visíveis (PRADO et al., 2012). Contudo, segundo os últimos autores, existem divergências quanto ao número de instares dessa espécie, as quais são atribuídas à dificuldade em diferenciá-los. A reprodução de $S$. oleae pode ocorrer de forma sexual, mas principalmente partenogenética (BARRANCO et al., 2008). As fêmeas de $S$. oleae podem colocar de 2.000 a 2.500 ovos, que permanecem incubados dentro destas, por cerca de 5 a 20 dias, dependendo das condições climáticas (NETO et al., 2008).

A dinâmica populacional da cochonilhanegra é conhecida em Portugal (PEREIRA et al., 1997; SANTA-CECÍlIA et al., 2014), Espanha (BRIALES;CAMPOS, 1986 ; MONTIEL;SANTAELLA, 1995; TENA et al., 2007; BARRANCO et al., 2008), Grécia (STRATOPOULOU; KAPATOS, 1998) e na Argentina (FERNANDEZ et al., 1979). No Brasil, os únicos trabalhos desta natureza foram os levantamentos populacionais realizados em pomares de oliveiras no município de Maria da Fé-MG, por Prado et al. (2012) e Santa-Cecília et al. (2014). No Rio Grande do Sul, onde o cultivo de oliveiras é recente, atualmente, a área de produção abrange 1,2 mil hectares (Emater/RS-Ascar, 2014), trabalhos que abordem a dinâmica populacional da cochonilha-negra inexistem. Este tipo de informação é fundamental para o aprimoramento do manejo da cultura, e desta forma este trabalho teve como objetivo conhecer a dinâmica populacional de $S$. oleae e quantificar seu parasitismo natural em oliveiras.

\section{MATERIAL E MÉTODOS}

O estudo foi realizado em Caçapava do Sul $\left(30^{\circ} 30^{\prime} 43^{\prime \prime} \mathrm{S}, 53^{\circ} 29^{\prime} 27^{\prime \prime}\right.$ O), Rio Grande do Sul, em pomar de oliveira variedade Arbequina, com 0,95 ha e 471 árvores, implantadas em 2006. Os registros meteorológicos diários de temperatura $\left({ }^{\circ} \mathrm{C}\right)$, precipitação pluviométrica $(\mathrm{mm})$ e umidade relativa do ar (\%) foram obtidos da Estação Meteorológica Automática de Caçapava do Sul, do Instituto Nacional de Meteorologia, localizada a $6,8 \mathrm{~km}$ da área de estudo. As amostragens foram mensais, de abril de 2012 a março de 2013, em 20 plantas sorteadas. De cada árvore, em cada um dos quadrantes da copa (norte, sul, leste e oeste), foram retirados dois ramos, sendo um da área externa e outro da interna, acerca de $1,50 \mathrm{~m}$ do solo, totalizando oito ramos por planta. Cada ramo (unidade amostral), com 20 a $30 \mathrm{~cm}$ de comprimento e, no mínimo, 20 folhas, foi acondicionado individualmente em saco plástico identificado, levado para o laboratório e mantido em refrigerador $\left( \pm 4{ }^{\circ} \mathrm{C}\right)$ até à a triagem, feita com o auxílio de estereomicroscópio. O local onde as cochonilhas se encontravam na unidade amostral foi registrado, face abaxial da folha (AB), adaxial (AD), borda (BO) e ramo (RA).

Os coccídeos foram examinados, separando o corpo do substrato, e contabilizados, por fase de desenvolvimento. Ninfas de primeiro e segundo instares $(\mathrm{N} 1+\mathrm{N} 2)$ foram contabilizadas juntas, por apresentarem características morfológicas semelhantes (corpo pequeno, achatado, tenro e amarelado) por difícil a separação . Registraram-se também ninfas de terceiro (N3), que apresentam coloração parda e uma marca em forma de $\mathrm{H}$ no corpo. Os adultos que têm coloração marrom-escura, corpo convexo e esclerotizado foram separados em sem ovos (A), e com ovos (AOV). Foram registrados indivíduos vivos $(\mathrm{V})$, mortos sem indícios de parasitismo $(\mathrm{M})$, parasitados com larvas de parasitoides (LA), com pupas de parasitoides (PU), com perfuração após a emergência de parasitoides (CP) e com mecônio, as fezes deixadas pelo parasitoide após a emergência (ME). 
O número médio de indivíduos em cada estágio de desenvolvimento, por árvore, em cada ocasião de amostragem e por estação, foi comparado por Kruskal-Wallis. O número médio de indivíduos nos ramos internos e externos, por árvore, foi comparado pelo teste de Mann-Whitney para duas amostras independentes. Foi comparada por Kruskal-Wallis a média de indivíduos por quadrante, por árvore e a média, por local, na unidade amostral ( $\mathrm{AB}, \mathrm{AD}, \mathrm{RA}$ ou $\mathrm{BO}$ ), assim como a média de cochonilhas mortas parasitadas (MP) e a de mortas não parasitadas (MNP). Para o cálculo da porcentagem de parasitismo, o número de indivíduos parasitados incluiu as cochonilhas $\mathrm{CP}, \mathrm{PU}, \mathrm{LA}$ e ME. O teste de correlação de Spearman foi realizado para avaliar a associação dos fatores meteorológicos com o número médio mensal de indivíduos, por árvore. O nível mínimo de significância adotado em todas as análises foi de $5 \%$, e o programa utilizado foi o Bioestat 5.0 $0^{\circledR}$ (AYRES et al., 2007).

\section{RESULTADOS E DISCUSSÃO}

O número médio de indivíduos por ocasião de amostragem no pomar foi de 755,4 $\pm 59,40$. Verificou-se que, embora $S$. oleae tenha ocorrido o ano todo, foi mais abundante na primavera $(3.543,8$ $\pm 378,25)$ e no verão $(4.279,3 \pm 346,89)$, quando comparados com o outono $(535,7 \pm 134,72)$ e o inverno $(705,7 \pm 162,84)(\mathrm{H}=54,2387 ; \mathrm{gl}=3$; $\mathrm{p}<0,0001$ ), sugerindo que as temperaturas mais elevadas destas duas estações são as mais favoráveis para este inseto. Tena et al. (2007), em citros e oliveira, na Espanha, também encontraram maior abundância de $S$. oleae no verão, assim como Marrão (2013), nos olivais em Portugal, corroborando os dados encontrados neste estudo. As temperaturas mínima ( $\mathrm{rs}=0,6375 ; \mathrm{p}=0,0257)$, média ( $\mathrm{rs}=$ $0,6538 ; \mathrm{p}=0,0211)$ e máxima $(\mathrm{rs}=0,6434 ; \mathrm{p}=$ $0,024)$ correlacionaram-se, de maneira positiva e significativa, com o número médio de indivíduos observados. Fernandez et al. (1979), na Argentina, e Jimenez (1985), na Espanha, afirmaram que, dentre os fatores climáticos, a temperatura é o principal agente de controle de ovos e ninfas de $S$. oleae em oliveiras. Da mesma forma, na Espanha, Montiel e Santaella (1995) constataram que a alta mortalidade de ninfas de primeiro instar da cochonilha-negra estava associada a temperaturas extremas (mínimas de $8{ }^{\circ} \mathrm{C}$ negativos; máximas de $40{ }^{\circ} \mathrm{C}$ e umidade relativa do ar de $30 \%$ ).

Por outro lado, em olivais, na Grécia, Stratopoulou e Kapatos (1998) apontaram que as altas temperaturas foram mais importantes que as baixas, como meio de controle abiótico de S. oleae. As temperaturas registradas em Caçapava do Sul (médias máximas de até $25{ }^{\circ} \mathrm{C}$ e mínimas de 10 ${ }^{\circ} \mathrm{C}$ ), embora com amplitude de variação pequena, mostraram associação com o número médio de cochonilhas. A umidade relativa do ar mantevese sempre acima de $70 \%$, não criando assim uma condição limitante no ambiente para as cochonilhas.

A ocorrência de indivíduos de S. oleae, nos diferentes estágios de desenvolvimento, em todas as ocasiões de amostragem, sugere que mais do que uma geração pode ocorrer ao longo do ano. A existência de mais de uma geração da cochonilha-negra ao longo do ano, com sobreposição das mesmas, foi constatada por Prado et al. (2012), em Minas Gerais, em oliveiras. Porém, em outras regiões, na Espanha (BRIALES; CAMPOS, 1986; MONTIEL; SANTAELLA, 1995) e em Portugal (PEREIRA et al., 1997), apenas uma geração anual tem sido registrada para a espécie. Constatou-se que, entre abril e outubro, o número médio mensal de ninfas de primeiro e segundo instares $(178,2 \pm 241,46)$ foi inferior (Fig. 1), em comparação ao observado entre novembro e março $(1.423,4 \pm 899,27)(\mathrm{H}=$ $131,3241 ; \mathrm{gl}=11 ; \mathrm{p}<0,0001)$. A diferença entre estes dois períodos representa um acréscimo de $571 \%$ no número de indivíduos.

$\mathrm{O}$ incremento das temperaturas, além da disponibilidade contínua de recurso alimentar pelas oliveiras, parece estimular a reprodução e a eclosão de ninfas, sugerindo ser este o principal período de recrutamento de novos indivíduos. Resultados diferentes foram observados na Espanha, em oliveiras, por Briales e Campos (1986) e Tena et al. (2007), os quais constataram que a eclosão das ninfas ocorreu concentrada durante junho e julho (início do verão). Segundo os autores, no final do verão, em função das altas temperaturas $\left(>30^{\circ} \mathrm{C}\right)$, associadas com umidade relativa inferior a $30 \%$, cerca de $80 \%$ das ninfas de $1^{\circ}$ instar morreram.

Temperaturas tão elevadas quanto às da Espanha não foram registradas em Caçapava do Sul, o que talvez explique a reprodução e a eclosão ao longo de toda esta estação. Quanto ao terceiro instar (N3) (Fig. 1), em média, o número não variou de abril a dezembro $(7,9 \pm 11,11)$ e foi mais elevado de janeiro até março $(69,9 \pm 48,83)(\mathrm{H}=116.6876$; $\mathrm{gl}=11 ; \mathrm{p}<0,0001)$. Briales e Campos (1986), na Espanha, encontraram predominância das fases imaturas, principalmente N2 e N3 durante o inverno, representando $75 \%$ da população neste período, embora também estivessem presentes durante todo o ano, porém em menor proporção. 
A média de adultos (A) de $S$. oleae (Fig. 1) foi mais baixa $(15,8 \pm 23,93)$ de abril a dezembro, em comparação ao período de janeiro a março $(46,4$ $\pm 35,57)(\mathrm{H}=54.8218 ; \mathrm{gl}=11 ; \mathrm{p}<0,0001)$. Adultos com ovos (AOV) (Fig. 1) seguiram o mesmo padrão dos sem ovos, de abril até dezembro $(6,3 \pm 9,70)$, sendo a média menor que a de janeiro a março $(22,1 \pm 16,26)(\mathrm{H}=80,2365 ; \mathrm{gl}=11 ; \mathrm{p}<0,0001)$. Carvalho e Aguiar (1997) também encontraram em citros, na Ilha da Madeira, adultos de $S$. oleae com e sem ovos, durante todo o ano. No entanto, por não terem conseguido detectar a longevidade dos indivíduos a campo, os autores não afirmaram se as fêmeas observadas ao longo do ano foram indivíduos de uma ou de mais gerações, e esta mesma dificuldade ocorreu no presente trabalho. O número de cochonilhas nos ramos internos e externos da copa não diferiu $(Z(U)=0,1086 ; p=0,4568)$, assim como entre os quadrantes norte, sul, leste e oeste $(\mathrm{H}=4,3468 ; \mathrm{gl}=3 ; \mathrm{p}=0,2264)$. Em oliveiras, em Portugal, Pereira et al. (2004) também não observaram diferença no número de $S$. oleae entre quadrantes da copa; no entanto, verificaram maior quantidade na região interna da mesma.

As ninfas $(\mathrm{N} 1+\mathrm{N} 2)$ foram mais abundantes na face abaxial das folhas $(\mathrm{H}=127,529 ; \mathrm{gl}=2 ; \mathrm{p}<$ 0,0001 ), fato que pode estar relacionado à proteção da luz solar. Conforme Carvalho e Aguiar (1997), no primeiro instar, os indivíduos apresentam reação fototrópica negativa. A busca por micro-hábitats mais abrigados da luz e úmidos na face abaxial das folhas, por ninfas neste estágio, foi referida em outros trabalhos (PEREIRA et al., 2004; SERAFINI et al., 2007; PRADO et al., 2012; SANTA-CECÍLIA et al., 2014). Afora as condições abióticas mais favoráveis, este micro-habitat oferece proteção contra a ação de inimigos naturais. Os resultados sugerem que, conforme avança o desenvolvimento, $S$. oleae direciona-se para os ramos, o que pode ser constatado pelo maior número de indivíduos dos demais estágios localizados nesta região; ninfas de terceiro instar $(\mathrm{H}=$ $107,0038 ; \mathrm{gl}=2 ; \mathrm{p}<0,0001)$, adultos $(\mathrm{H}=280,842 ; \mathrm{gl}$ $=2 ; \mathrm{p}<0,0001)$ e adultos com ovos $(\mathrm{H}=206,1361$; $\mathrm{gl}=2 ; \mathrm{p}<0,0001$ ) (Fig. 2).

A maior abundância de N1 + N2 nas folhas também pode ser devido ao aparelho bucal mais delicado; sendo o tecido foliar mais tenro que o dos ramos, facilitaria a alimentação. Os ramos constituem-se em recurso mais perene na planta, e neste local registrou-se o maior número de N3, A e AOV, possivelmente por estes serem os estágios mais longos no desenvolvimento. Resultados semelhantes foram obtidos por Tena et al. (2007), que constataram $75 \%$ das ninfas de primeiro instar nas folhas de oliveira e apenas 5\% de adultos. Prado et al. (2012) e Santa-Cecília et al. (2014) também observaram esta migração das folhas para os ramos, conforme o desenvolvimento dos indivíduos de $S$. oleae.

Quanto à média de indivíduos de $S$. oleae mortos não parasitados (MNP) e mortos parasitados (MP), esta diferiu entre os meses $(\mathrm{H}=68,659$; $\mathrm{gl}=$ $11 ; p<0,0001)$. Entre abril e novembro, a proporção de MNP foi, no mínimo, nove vezes maior que o de MP (Fig. 3). Em dezembro, o número de MP foi equivalente ao de MNP. A partir de janeiro até março, o número de MNP voltou a subir e foi superior ao de MP. Embora, Stratopoulou e Kapatos (1998) tenham afirmado que a mortalidade por parasitismo pode ser importante para a regulação da população de $S$. oleae, neste estudo, este não foi o principal fator.

Segundo observaram Montiel e Santaella (1995), além de inimigos naturais e fatores climáticos, a competição intraespecífica também foi responsável pela mortalidade da cochonilha-negra. Estes autores constataram que, nos meses de verão, ninfas de $S$. oleae morreram à procura por lugares mais favoráveis para se fixar e alimentar-se, permanecendo mais expostas às altas temperaturas. Assim, é possível que a mortalidade observada nas oliveiras, em Caçapava do Sul, tenha sido decorrente, principalmente, da competição intraespecífica, pois $90 \%$ dos indivíduos $\mathrm{N} 1+\mathrm{N} 2$ estavam mortos. O parasitismo de S. oleae mais elevado no mês de dezembro tanto pode estar associado às condições climáticas favoráveis ao desenvolvimento dos parasitoides, quanto à maior abundância do hospedeiro. Corroborando estes resultados, maior abundância de parasitoides de S. oleae foi verificada no verão, em Chipre, na Espanha, assim como em Minas Gerais, no Brasil (ORPHANIDES, 1993; TENA et al., 2008; PRADO et al., 2012).

Saissetia oleae apresentou a taxa de parasitismo de $3,40 \%$, e deste total, $49,2 \%$ são de cochonilhas perfuradas (CP) e $50 \%$ de pupas de parasitoides (PU). Este resultado é inferior ao observado em Portugal (9,5\%) e em Minas Gerais, Brasil (32,5\%) (PEREIRA et al., 1998; PRADO et al., 2012). No entanto, estes autores consideraram baixo o parasitismo na cochonilha-negra. A taxa de parasitismo pode ser subestimada pela mortalidade da população hospedeira em decorrência das puncturas realizadas pelos parasitoides para alimentação e para avaliação do hospedeiro, as quais podem ter contribuição importante para a mortalidade total (ASPLANATO; GARCÍA, 2002); no entanto, no presente trabalho, estas não foram observadas $\mathrm{e}$ contabilizadas. 


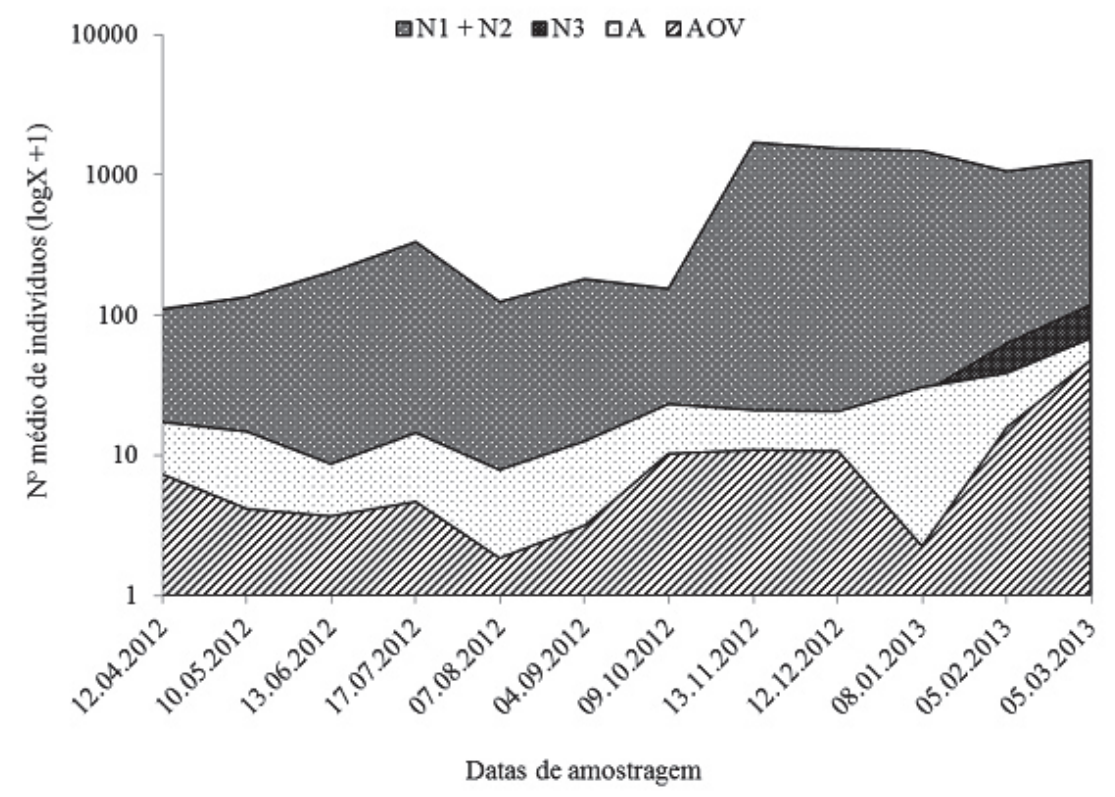

FIGURA 1 - Número médio de indivíduos de Saissetia oleae, por árvore, em cada ocasião de amostragem, na fase de ninfa de primeiro e segundo instares (N1+N2), ninfa de terceiro instar (N3), adultos (A) e adultos com ovos (AOV). Caçapava do Sul-RS, de abril de 2012 a março de 2013.

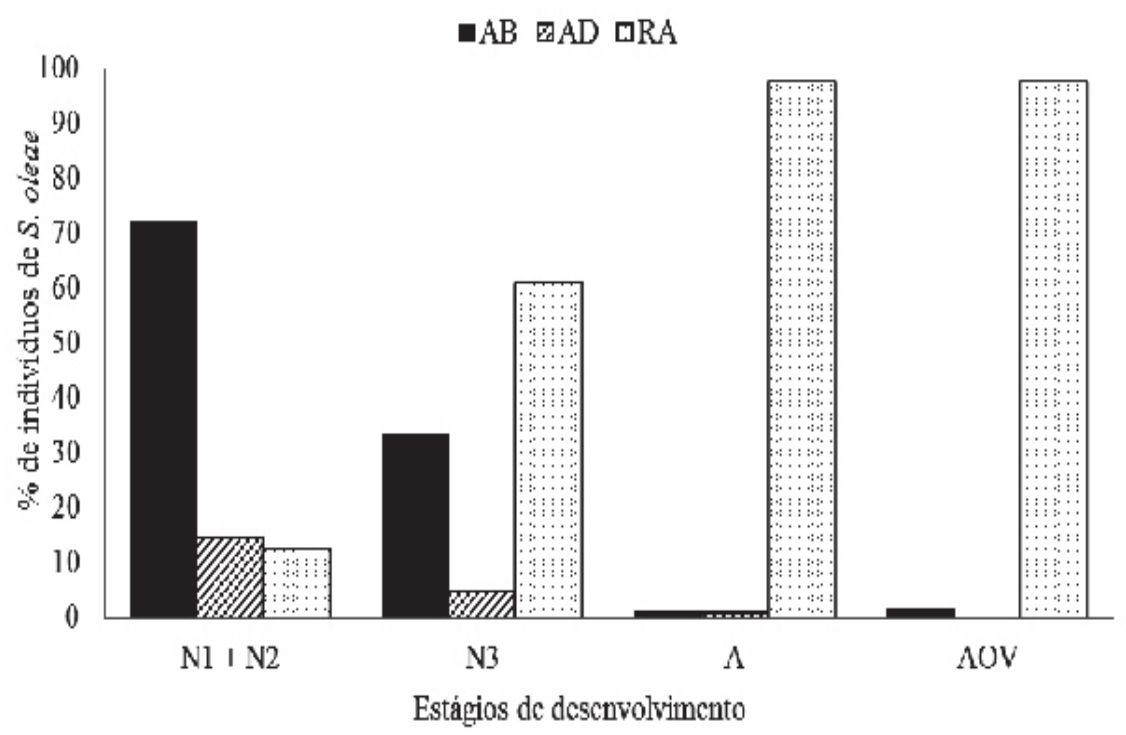

FIGURA 2 - Porcentagem (\%) de indivíduos de Saissetia oleae nos estágios: ninfa 1 e 2 (N1+N2), Adultos (A) e Adultos com ovos (AOV), amostrados nos ramos (RA), na face abaxial (AB) e adaxial (AD) de folha em oliveiras. Caçapava do Sul-RS, de abril de 2012 a março de 2013. 


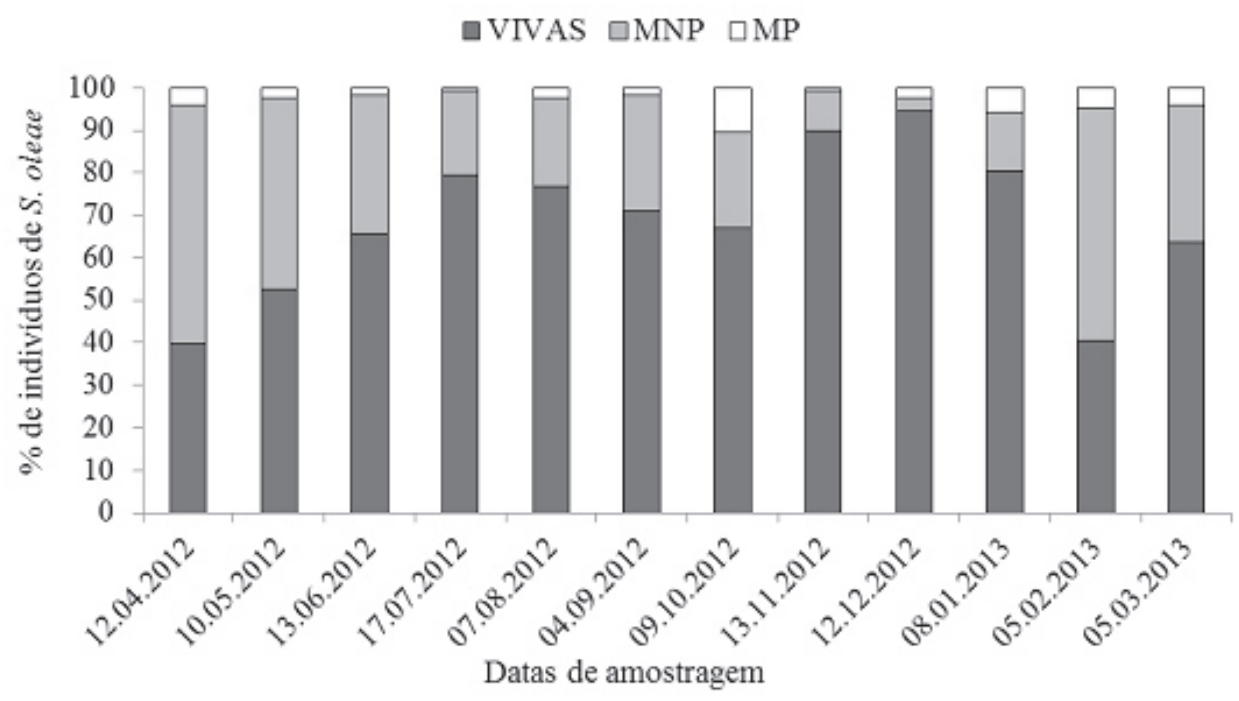

FIGURA 3 - Porcentagem de indivíduos de Saissetia oleae, vivos (VIVAS), mortos não parasitados (MNP) e mortos parasitados (MP), por ocasião de amostragem em oliveiras. Caçapava do Sul-RS, de abril de 2012 a março de 2013.

\section{CONCLUSÕES} abundante.

Na primavera e no verão, S. oleae é mais

A temperatura é o fator meteorológico que mais afeta o tamanho da população da cochonilhanegra.

Ninfas de primeiro e segundo instares são mais frequentes nas folhas de oliveiras, enquanto as de terceiro e os adultos são nos ramos.

A taxa de parasitismo é de 3,40\%.

\section{AGRADECIMENTOS}

À CAPES, pela concessão de bolsa à primeira autora, e ao CNPq, para a segunda autora (Processo $\left.\mathrm{n}^{\circ} 303606 / 2013-4\right)$, e para a terceira autora, pela bolsa de Pós-Doutorado Empresarial (PDI).

\section{REFERÊNCIAS}

ASPLANATO G.; GARCÍA M. Ciclo estacional de la cochinilla roja californiana, Aonidiella aurantii (Maskell) (Homoptera: Diaspididae) em naranjos del sur de Uruguay. Agrociencia, Montecillo, v. 5, n. 1, p. 54-67, 2001.
AYRES, M.; AYRES, M. Jr; AYRES, D. L.; DOS SANTOS, A. S. BioEstat 5.0 aplicações estatísticas nas áreas da ciências biológicas e médicas. Belem: Sociedade Civil Mamiraua/CNPq, 2007. 324 p.

BARRANCO, D.; FERNÁNDEZ-ESCOBAR, R.; RALLO, L. El cultivo del olivo. 6. ed. Madrid: Ediciones Mundi Prensa y Junta de Andalucía, 2008. $846 \mathrm{p}$.

BRIALES, M. J.; CAMPOS, M. Estudio de la biología de Saissetia oleae (Olivier, 1791) (Hom. Coccidae) em Granada (España). Boletín de la Asociación Española de Entomología, Salamanca, v. 10, p. 249-256, 1986.

CARVAlHO, J. P.; AGUIAR, A. M. F. Pragas dos citros na Ilha da Madeira. Funchal: Secretaria Regional de Agricultura, Florestas e Pescas 1997. p. 238-248.

EMATER- Empresa de Assistência Técnica e Extensão Rural do Rio Grande do Sul. Associação de crédito e assistência rural. 2014. Disponível em: $\leq \mathrm{http} / / / \mathrm{www} . \mathrm{emater}$.tche.br/site/noticias/detalhenoticia.php?id=19781\#.VQrdCNLF-VM $>$. Acesso em: 19 de mar. 2015. 
FERNANDEZ, J. M.; MENDIVIL, Z.; ALMAGRO, F. Estudio de Saissetia oleae en Córdoba. Boletin del Servicio de Plagas Forestales, Madrid, v. 5, p. 149-156, 1979.

JIMENEZ, A. Potential value of entomophagous in the olive pests control. In: THE CEC /FAO/ IOBC INTERNATTONAL JOINT MEET, 1985, Pisa. Proceedings... Boston: Commission of the European Communities, 1985. p. 441-450.

MARRÃO, R. M. Influência do tamanho do hospedeiro no parasitismo exercido sobre Saissetia oleae (Olivier). 2013. 103 f. Dissertação (Mestrado) - Escola Superior Agrária de Bragança, Bragança, 2013.

MONTIEL, A.; SANTAELLA, S. Evolución de la población de Saissetia oleae (Oliv.) en condiciones naturales. Períodos susceptibles de control biológico. Boletín de Sanidad Vegetal. Plagas, Madrid, v. 21, p. 445- 455, 1995.

MURÚA, M. G.; FIDALGO, P. Listado preliminar de los enemigos naturales de Saissetia oleae (Homoptera: Coccidae) em olivares de la provincia de La Rioja, Argentina. Boletín de Sanidad Vegetal. Plagas, Madrid, v. 27, p. 447-454, 2001.

NETO, J. V.; OLIVEIRA, A. F.; OLIVEIRA, N. C.; DUARTE, H. S. S.; GONÇALVES, E. D. Aspectos técnicos da cultura da oliveira. Belo Horizonte: EPAMIG, 2008. 56 p. (Boletim Técnico, 88)

ORPHANIDES, G. M. Control of Saissetia oleae (Hom.:Coccidae) in Cyprus through establishment of Metaphycus barletti and M. Helvolus (Hym. : encyrtidae). Entomophaga, Paris, v. 38, n. 2, p. 235-239, 1993.

PEREIRA J. A. C. Bioecologia da cochonilha negra, Saissetia oleae (Olivier), em oliveira, em Trás-os-Montes. 2004. 121 f. Tese (Doutorado) Universidad de Trás-os-Montes e Alto Douro, Vila Real, Portugal. 2004.

PEREIRA, J. A.; TORRES, L.; CABANAS, J. E.; BENTO, A. Parasitismo associado a Saissetia oleae (Oliv.) em Trás-os-Montes. Revista de Ciências Agrárias, Lisboa, v.21, p. 237-244, 1998.
PEREIRA, J.; CARREIRA, F.; BENTO, A.; ESPINHA, I.; TARRES, L. Ciclo biológico da cochonilha negra, Saissetia oleae (Olivier), em oliveira, na Terra Quente Transmontana. In: ENCONTRO NACIONAL DE PROTEÇÃO INTEGRADA, 4., 1997, Ponta Grossa. Anais... p. 245-249.

PRADO E. C.; SANTA-CECÍLIA, L. V. C.; ALVARENGA, T. M. Pragas da oliveira: Bioecologia, inimigos naturais e manejo. In: OLIVEIRA, A.F. Oliveira no Brasil tecnologias de produção. Belo Horizonte: Empresa de Pesquisa Agropecuária de Minas Gerais, 2012. p.498 -515.

SANTA-CECÍLIA, L. V. C; PRADO, E.; PEREIRA, A. B. Cochonilha-preta da oliveira: bioecologia e controle. Belo Horizonte: Epamig, 2014. (Circular Técnica, 194)

SERAFINI, F.; SBITRI, M. O.; TOMBESI, A.; TOMBESI, S.; SAAVEDRA,M. S.; ESCOBAR, R. F. Técnicas de producción en olivicultura. Madrid: Consejo Oleícola Internacional, 2007.

STRATOPOULOU, E. T.; KAPATOS, E. T. Key factors and regulation of population of Saissetia oleae (Horn., Coccidae) on olive trees in the region of Magnesia, Greece. Journal of Applied Entomology, Berlin, v. 122, p. 501-507, 1998.

TENA, A.; SOTO, A.; GARCIA-MARÍ, F. Parasitoid complex of black scale Saissetia oleae on citrus and olives: parasitoid species composition and seasonal trend. BioControl, Dordrecht, v. 53, n. 3, p.473-487, 2008.

TENA, A.; SOTO, A.; VERCHER, R.; GARCIAMARÍ, F. Density and structure of Saissetia oleae (Hemiptera: Coccidae) populations on citrus and olives: relative importance of the two annual generations. Environmental Entomology, College Park, v. 36, n. 4, p. 700-706, 2007. 